\title{
Deglutition evaluation with high density surface electromyography in post-irradiated nasopharyngeal cancer patients with dysphagia
}

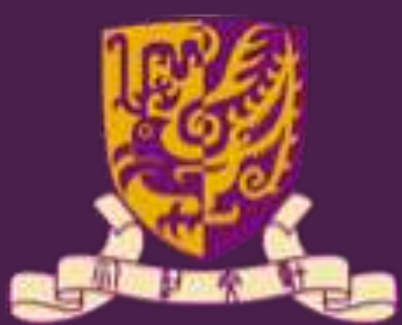

and 8

\author{
Ms. Ka Ying Karman Leung, Professor Michael Chi Fai Tong \\ Department of Otorhinolaryngology, Head and Neck Surgery \\ The Chinese University of Hong Kong
}

\section{BACKGROUND}

Various methods have been applied to study physiological aspects of swallowing, including fiberoptic endoscopic evaluation of swallowing (FEES) and videofluoroscopic swallowing study (VFSS). Investigation of the dynamics of the swallowing muscles during swallowing process has been limited. High density surface electromyography(HD-sEMG) is a non-invasive technique to evaluate muscle function defects. A novel methodology of deglutition evaluation with HD-sEMG was described and investigated in normal subjects. $(1,2)$

\section{OBJECTIVES}

The objective of the study is to investigate the deglutition evaluation with HD-sEMG in post-irradiated nasopharyngeal carcinoma(NPC) patients with dysphagia.

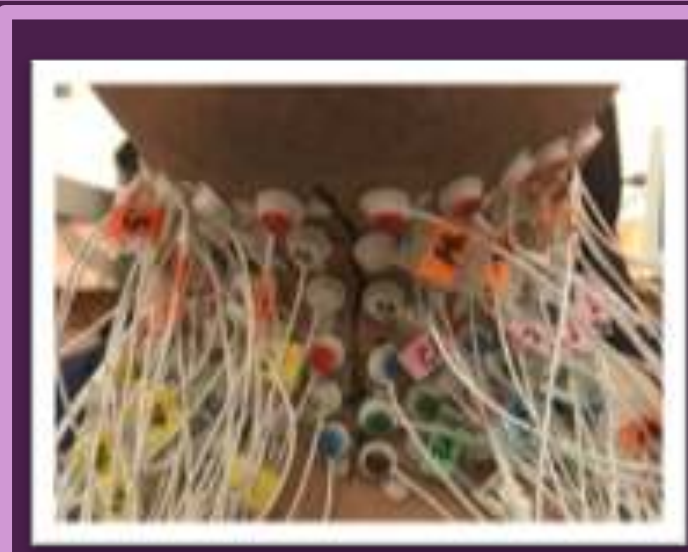

Figure1. HD-SEMG Electrode array alignment and placement on anterior neck

\section{METHODS}

The HD-sEMG signals were recorded from 5 healthy subjects and 5 post-irradiated NPC patients with dysphagia during different swallowing tasks. The electrodes were placed on the anterior neck of each subject in $16 \mathrm{x}$ 6 array(Figure.1). Each subject was required to swallow 5 trials $\times 5 \mathrm{ml} \times 5$ different consistency of food including thin liquid (water), thick liquid, congee, puree, gastric rice

The HD-sEMG recordings were segmented into a series of $100 \mathrm{~ms}$ analysis windows. The root mean square (RMS) of the 96channel sEMG signals was calculated for each analysis window to represent the averaged power of the signals. A dynamic spatial 2D color topographic map of muscle activities throughout the entire swallow process was generated with RMS value in $16 \times 6$ array and the averaged power of sEMG signals was presented using different color tones (cold to hot color).

\section{CONCLUSIONS}

The mapping provided detailed spatial information of neck muscle activities during swallowing. Deglutition evaluation with HDsEMG is promising and capable for further refinement for clinical assessment of patients with dysphagia.

\section{RESULTS}

Dynamic topography was generated from the root mean square of the HD-sEMG signals to illustrate the neck muscle function in the whole swallowing process(Figure. 2)(Video). The dynamic topography showed different swallowing patterns between the dysphagic (right) and healthy (left) individuals.

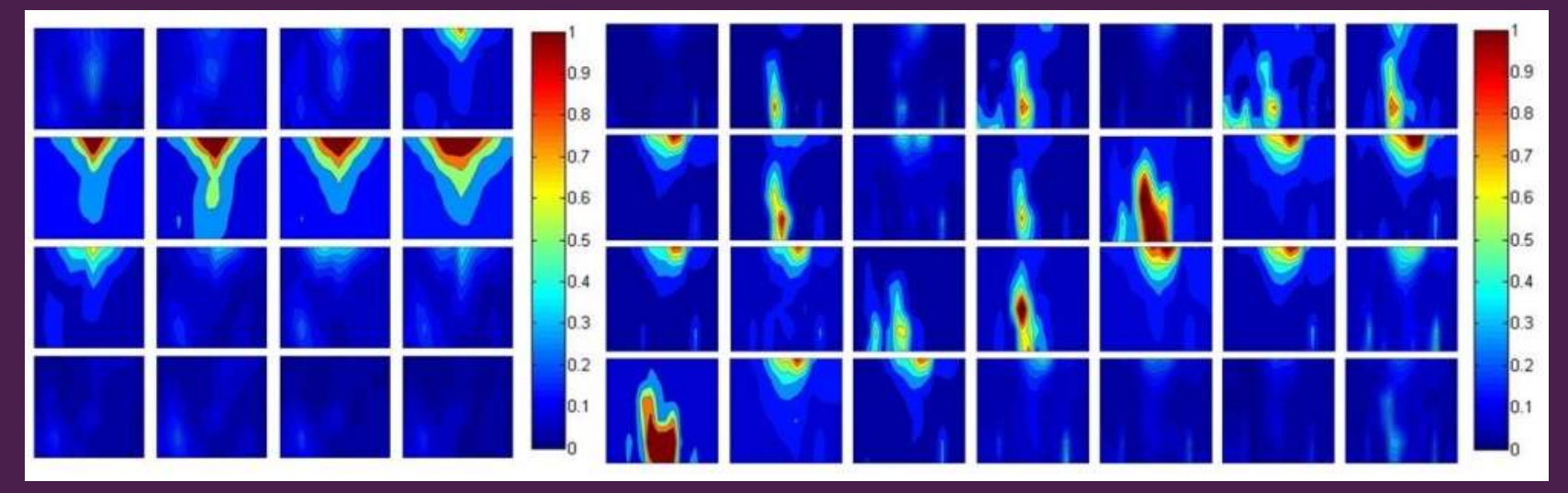

Figure 2. Surface EMG topography as a representation of muscle activation swallowing $5 \mathrm{~m}$ water in a healthy subject (left) and a dysphagia patient with bilateral tongue atrophy and poor jaw movement, velopharyngeal competence and neck movement.

A symmetric pattern of the mean EMG signals was shown in video (Left) suggesting that the neck muscle contraction during swallow was symmetric in healthy individuals. The EMG signals were gradually weaker from the middle to the side and also from the top to the bottom suggesting that suprahyoid muscles contraction played a critical role in swallowing rather than infrahyoid muscle group. The dynamic topography showed a symmetric and triangular pattern. The whole process of a single swallow shown in dynamic topography was smooth and coherent.

A post-irradiated nasopharyngeal carcinoma patient aged 54 was suffering from dysphagia with bilateral tongue atrophy and poor jaw movement, velopharyngeal competence and neck movement. The signals in the patient were much stronger than healthy individuals. Multiple swallows were performed. The pattern was asymmetric. The dynamic topography showed the suprahyoid muscles contraction was followed by the infrahyoid muscles contraction which was much stronger in the patient compared to healthy individuals. The muscles contraction was stronger to compensate the muscle defects. Suprahyoid muscles were the main muscle group contributing to swallow in normal individuals while in the patient with muscles defects, both suprahyoid muscles and infrahyoid muscles contributed to the swallow process.

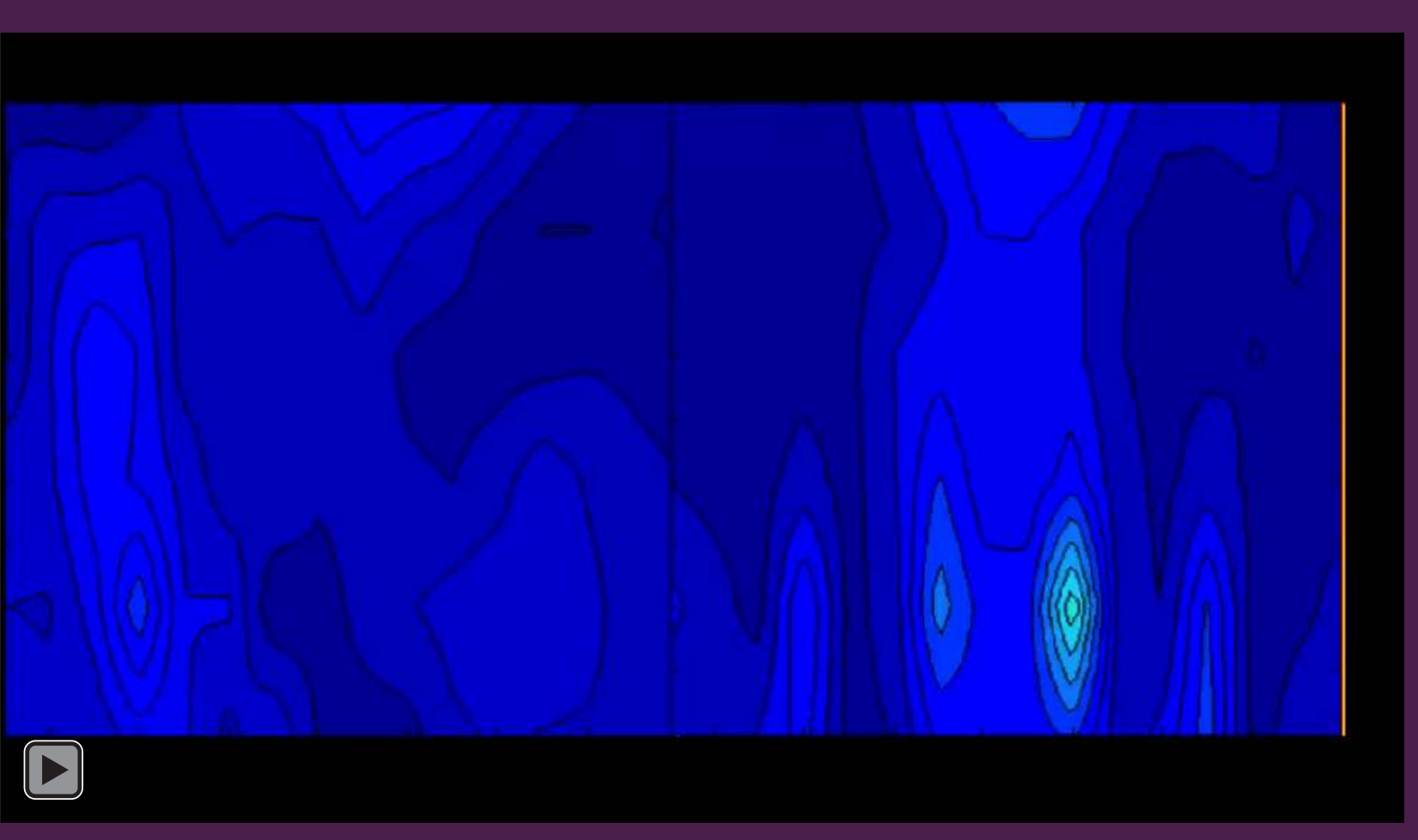

Suprahyoid muscle group

Hyoid bone

Infrahyoid muscle group

Video: Dynamic topography of healthy individual swallowing $5 \mathrm{ml}$ of water(Left). Dynamic topography of dysphagic individual swallowing $5 \mathrm{ml}$ of water (Right).

1. Bin Yu et al., 2013. A pilot study of high-density electromyographic maps of muscle activity in normal deglutition. Engineering in Medicine and Biology Society (EMBC), 2013 35th Annual International Conference of the IEEE, pp.6635-6638. 